\title{
Spiral Exchange Interaction in Diluted Magnetic Semiconductor Junction
}

\author{
Shih-Jye Sun \\ Department of Applied Physics, National Chia-Yi University, Chia-Yi 600, Taiwan \\ Song-Shien Cheng \\ Department of Physics, National Tsing-Hua University, Hsinchu 300, Taiwan \\ Hsiu-Hau Lin \\ Department of Physics, National Tsing-Hua University and Physics Division, \\ National Center for Theoretical Science, Hsinchu 300, Taiwan
}

(Dated: November 19, 2018)

\begin{abstract}
We studied the exchange interactions mediated by polarized itinerant carriers in diluted magnetic semiconductor (DMS) junction. In contrast to the ordinary RKKY oscillations, the induced moment possesses an interesting spiral motion, accompanied with angular oscillations. The spiral motion remains robust in the entire $T<T_{c}$ regime while the oscillatory motion gets enhanced as $T \rightarrow T_{c}$. To explore the novel phenomena, we propose a ferromagnet/DMS/ferromagnet junction would bring out interesting spin-dependent transport properties and possible applications in spintronics.
\end{abstract}

One of the key elements in spintronics is the diluted magnetic semiconductor (DMS), which incorporate both charge and spin phenomena together in one material 1, 2]. With rapid and intensive research attentions, robust ferromagnetic order was demonstrated in $\left(\mathrm{Ga}_{1-x} \mathrm{Mn}_{x}\right)$ As with Currie temperature around 150 $\mathrm{K}$ 3, 4]. It is generally believed that the ferromagnetism in DMS is mediated by the itinerant carriers in the host semiconductor [5, 6, 7, 8]. For the unpolarized itinerant carriers, the mediated exchange coupling among magnetic moments is the well-known RKKY interaction. However, since itinerant carriers in DMS is polarized with sensitive temperature dependence [9, 10, 11], it is interesting to study what kind of exchange coupling gets generated.

In this Letter, we employ the self-consistent Green's function approach to address this issue. Note that, while the RKKY exchange interaction is known for a long time, its direct experimental evidence is only detected recently 12]. An easier way is through the spindependent scattering in the ferromagnet/nonmagnetic metallic junction 13, 14]. Here we proposed a similar experimental setup, as shown in Fig. 1, to explore the effective exchange interaction in DMS. One might expect the effective exchange interaction mediated by itinerant carriers in DMS also exhibits similar RKKY-type behavior. However, we found the presence of finite polarization gives rise to qualitatively different behavior. In the ferromagnet/DMS/ferromagnet junction, the induced polarization is spiral with angle $\theta_{s}(d)$ depending on the width of the junction. The spatial dependence of the spiral angle at different temperatures will be studied in details later.

The DMS is modeled by the Hamiltonian, containing the kinetic energy of the itinerant carriers and the exchange interaction between itinerant carriers and the lo-

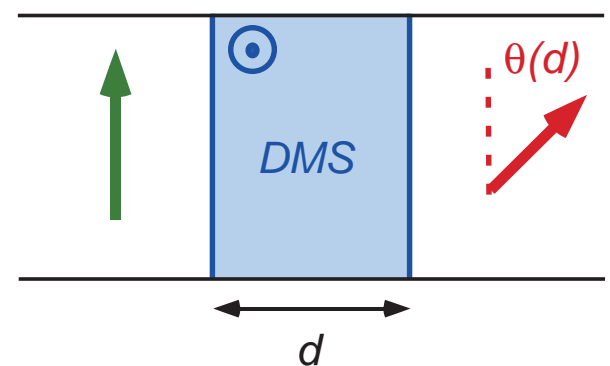

FIG. 1: Schematic diagram for a ferromagnet/DMS/ferromagnet junction. The polarization of DMS is placed in the $z$-direction and the magnetization on the left is fixed in $y$-direction. The induced polarization on the right has a spiral angle $\theta(d)$ depending on the junction width $d$.

calized moments doped into the host semiconductor,

$$
H=H_{0}+J \int d^{3} r \boldsymbol{S}(r) \cdot \boldsymbol{s}(r),
$$

where the spin density of the localized moments is $\boldsymbol{S}(r) \sum_{I} \delta^{3}\left(r-R_{I}\right) \boldsymbol{S}_{I}$ and the itinerant spin density is $\boldsymbol{s}(r)=\psi^{\dagger}(r)(\boldsymbol{\sigma} / 2) \psi(r)$. The band structure of the itinerant carriers is described by the host semiconductor. Since our emphasis here is to demonstrate the qualitative difference from the usual RKKY exchange coupling, the dispersion is taken to be the simplest parabolic band $H_{0}=p^{2} / 2 m^{*}$. Generalization to more realistic but complex band structures, such as the six-band Luttinger model, can be achieved straightforwardly. Throughout the Letter, the parameters would be set to the typical values in $\left(\mathrm{Ga}_{1-x}, \mathrm{Mn}_{x}\right)$ As: $J=0.15 \mathrm{eV} \mathrm{nm}{ }^{3}$ and $m^{*}=0.5 m_{e}$. Besides, the densities of itinerant carriers and localized moments are $c^{*}=0.1 \mathrm{~nm}^{-3}$ and $c=1$ $\mathrm{nm}^{-3}$ respectively.

The induced exchange interaction $J_{i j}(r)$ mediated by polarized itinerant carriers is directly proportional to the 


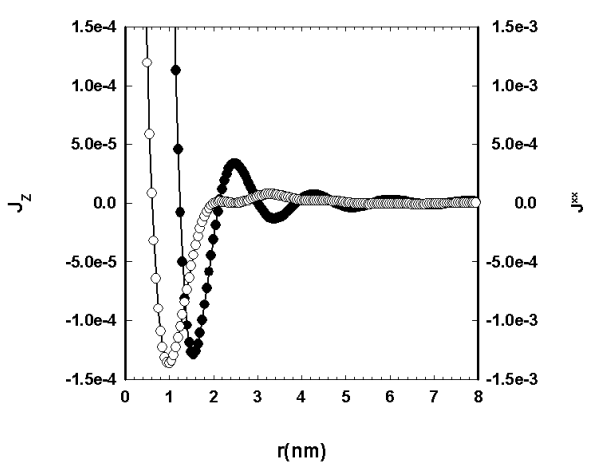

FIG. 2: The diagonal parts of exchange interactions $J_{z z}(r)$ (filled symbols) and $J_{x x}(r)$ at $T=10 K$.

static spin susceptibility, $J_{i j}(r)=J^{2} \chi_{i j}(r, \omega=0)$, within the linear-response regime. The spin susceptibility is

$$
\chi_{i j}(r, t)=-i \Theta(t)\left\langle\left\langle\left[s_{i}(r, t), s_{j}(0,0)\right]\right\rangle\right\rangle,
$$

where the double brackets implies both thermal and quantum averages. To compute the susceptibility at finite temperature, we employed the self-consistent Green's function method developed previously [15, 16] to obtain the propagators for itinerant carriers and localized moments. With these Green's functions, the average spin densities of itinerant and localized spins, $\left\langle S_{z}\right\rangle$ and $\left\langle s_{z}\right\rangle$ can be determined self-consistently in numerics. With the particular set of parameters we choose in this Letter, the Curie temperature is about $T_{c} \approx 45 \mathrm{~K}$.

Making use of Eq. 2 from employing equation of method on it [17], the exchange couplings generated from the diagonal parts of the spin susceptibility are

$$
\begin{aligned}
J_{z z}(r) & =\frac{J^{2} m}{4 \pi^{3} \hbar^{2}} \frac{1}{r^{2}} \int_{0}^{\infty} k \sin (k r) \cos (k r)\left(f_{\uparrow}+f_{\downarrow}\right) d k,(3) \\
J^{x x}(r) & =\frac{J^{2} m}{4 \pi^{3} \hbar^{2}} \frac{1}{r^{2}}\left[\int_{0}^{\infty} k \sin (k r) \cos \left(\sqrt{k^{2}+k_{\Delta}^{2}} r\right) f_{\downarrow} d k\right. \\
& +\int_{k_{\Delta}}^{\infty} k \sin (k r) \cos \left(\sqrt{k^{2}-k_{\Delta}^{2}} r\right) f_{\uparrow} d k \\
& \left.+\int_{0}^{k_{\Delta}} k \sin (k r) e^{-\sqrt{k_{\Delta}^{2}-k^{2}} r} f_{\uparrow} d k\right]
\end{aligned}
$$

where $\hbar^{2} k_{\Delta}^{2} / 2 m^{*}=\Delta$ and $\Delta=J\left\langle S_{z}\right\rangle$ is the effective Zeeman gap caused by the average local magnetization. The Fermi-Dirac distribution for spin-up and spin-down itinerant carriers are $f_{\uparrow, \downarrow}$ respectively.

The spatial dependences of $J_{z z}(r)$ and $J_{x x}(r)=J_{y y}(r)$ are shown in Fig. 2. It is evident that they show oscillatory RKKY behavior and decay quickly from the origin. Note that the finite polarization causes the non-uniform decay of the oscillation amplitudes. The finite polarization also gives rise to the off-diagonal exchange interactions. It is straightforward to show that $J_{z x}=J_{z y}=0$

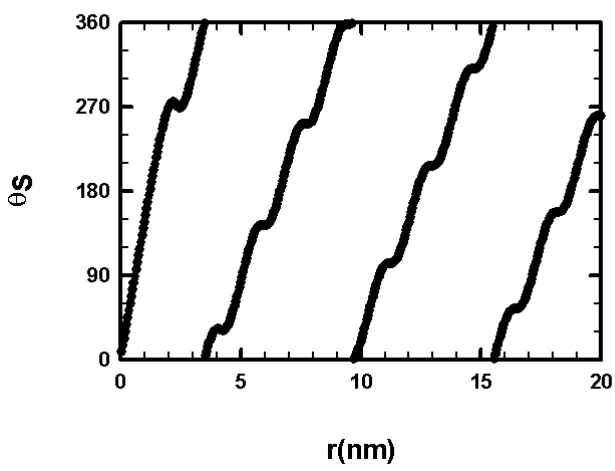

FIG. 3: The spiral angle $\theta_{s}(r)$ at $T=10 \mathrm{~K}$. The chemical potential only cut across the single band of one particular spin flavor and the polarization of itinerant carriers is almost one.

from symmetry arguments. The only non-vanishing offdiagonal exchange interaction is

$$
\begin{aligned}
J^{x y}(r) & =\frac{J^{2} m}{4 \pi^{3} \hbar^{2}} \frac{1}{r^{2}} \int_{k_{\Delta}}^{\infty} k \sin (k r) \sin \left(\sqrt{k^{2}-k_{\Delta}^{2}} r\right) f_{\uparrow} d k \\
& -\int_{0}^{\infty} k \sin (k r) \sin \left(\sqrt{k^{2}+k_{\Delta}^{2}} r\right) f_{\downarrow} d k .
\end{aligned}
$$

It is clear that the first and second integrals cancel when the polarization vanishes $k_{\Delta}=0$ and $f_{\uparrow}=f_{\downarrow}$. The spatial dependence of $J_{x y}(r)$ is also oscillatory as the diagonal parts. However, the existence of the off-diagonal exchange interaction gives rise an interesting phenomena which is absent for usual RKKY interaction.

Suppose we sandwich DMS with two ferromagnets as shown in Fig. 1. The perpendicular polarization between DMS and the left ferromagnet could be made by utilizing the anisotropy of DMS making the hard axis of DMS parallel to the left ferromagnet. The moment on the left ferromagnet would induce magnetization in both $y$ - and $x$-axes since the off-diagonal part is not zero. It is useful to define the spiral angle of the induced magnetic moment,

$$
\tan \theta_{s}(r)=\frac{J_{x y}(r)}{J_{y y}(r)} .
$$

The spatial dependences of the spiral angle at $T=10$ $\mathrm{K}$ and $T=44 \mathrm{~K}$ are plotted in Figure 3 and 4 . It is rather interesting that the induced moments through the DMS junction is characterized by both spiral rotations and angular oscillations. After some algebra, the corresponding length scales of spiral and oscillatory motions are approximately

$$
\begin{aligned}
& \lambda_{s} \approx \frac{2 \pi}{\sqrt{k_{F}^{2}+k_{\Delta}^{2}}-k_{F}}, \\
& \lambda_{o} \approx \frac{2 \pi}{\sqrt{k_{F}^{2}+k_{\Delta}^{2}}+k_{F}},
\end{aligned}
$$




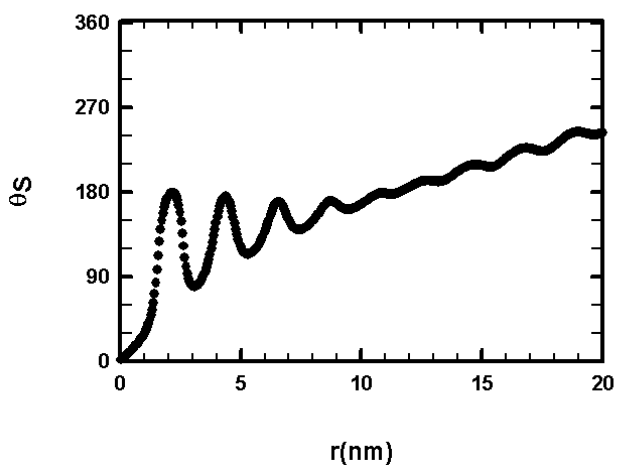

FIG. 4: The spiral angle $\theta_{s}(r)$ at $T=44 \mathrm{~K}$. Both bands of different spins are almost degenerate and the spin polarization is small.

where $k_{F}$ is the Fermi momentum of the unpolarized itinerant carriers.

At low temperature $T=10 \mathrm{~K}$, the chemical potential only cuts through single band and most of the itinerant carriers have the same spin orientation. The direction of the induced moment points along the direction of the spiral angle $\theta_{s}$, depending on the width of the DMS junction. As is clear from Fig. 3, accompanied with the spiral motion, there is also the oscillatory motion (with shorter period) and creates the wiggles in the trace. A remarkable feature is that the spiral motion does not seems to decay away as quickly as the exchange couplings $J_{x x}(r)$ and $J_{x y}(r)$ themselves. This may imply that the spiral dependence is easier to observe in experiments than the usual RKKY oscillations. Besides, the spiral-angular dependence of the induced moment also makes it charming for spin-dependent transport in tunnelling magnetoresistance (TMR) application and open up a new window for new functionality of device designs.

Close to the Currie temperature $T=44 \mathrm{~K}$, both bands of the itinerant carriers are almost degenerate and the polarization is small. From Eq. [7] we expect that the period of spiral motion become large, as shown in Fig. 4. The oscillatory motion becomes transparent within one spiral period and decays away rapidly. While one might naively expect that the spiral motion is weaken by the small polarization, it is actually not the case. The spiral motion persists but with a longer period. As the temperature approaches the critical one, the period of the spiral motion becomes infinitely long while the oscillatory motion gets enhanced. Eventually, the spiral angle only takes on discrete values, either $\theta_{s}=0$ or $\theta_{s}=\pi$, which correspond to the ordinary RKKY oscillations. However, as long as $T<T_{c}$, the mediated exchange interaction is qualitatively different from that generated by unpolarized itinerant carriers.

In conclusion, we studied the exchange interactions mediated by polarized itinerant carriers in DMS junction. We found, in contrast to the ordinary RKKY oscillations, the induced moment possesses a spiral motion accompanied with oscillatory motions. The spiral motion remains robust in the entire regime below the Curie temperature, while the angular oscillations get enhanced as the polarization becomes small. A ferromagnet/DMS/ferromagnet junction would bring out this novel spiral dependence and leads to interesting spindependent transport phenomena.

We thank the support of National Science Council in Taiwan through grants NSC-91-2112-M-230001(SJS), NSC-90-2112-M-007-050(HHL), NSC-91-2112M-007-040(HHL) and NSC-91-2120-M-007-001(HHL). The hospitality of the National Center for Theoretical Science, where the work was initiated, is greatly acknowledged.

[1] H. Ohno, Science 281, 951 (1998).

[2] G. A. Prinz, Science 282, 1660 (1998).

[3] K. C. Ku, S. J. Potashnik, R. F. Wang, S. H. Chun, P. Schiffer, N. Samarth, M. J. Seong, A. Mascarenhas, E. Johnston-Halperin, R. C. Myers, A. C. Gossard, and D. D. Awschalom, Appl. Phys. Lett. 82, 2302 (2003).

[4] K. W. Edmonds, K. Y. Wang, R. P. Campion, A. C. Neumann, N. R. S. Farley, B. L. Gallagher, and C. T. Foxon , Appl. Phys. Lett. 81, 4991 (2002).

[5] H. Akai, Phys. Rev. Lett. 81, 3002 (1998).

[6] J. König, H.-H. Lin and A. H. MacDonald, Phys. Rev. Lett. 84, 5628 (2000).

[7] J. Schliemann, J. König, H.-H. Lin and A. H. MacDonald, Appl. Phys. Lett. 78, 1550 (2001).

[8] V. I. Litvinov and V. K. Dugaev, Phys. Rev. Lett 86, 5593 (2001).

[9] H. Ohno, A. Shen, F. Matsukura, A. Oiwa, A. Endo, S. Katsumoto, and Y. Iye, Appl. Phys. Lett. 69, 363 (1996); H. Ohno, J. Magn. Magn. Mater. 200, 110 (1999).

[10] Y. Matsumoto, M. Murakami, T. Shono, T. Hasegawa, T. Fukumura, M. Kawasaki, P. Ahmet, T. Chikyow, S. Y. Koshihara, and H. Koinuma, Science 291. 854 (2001).

[11] T. Dietl, H. Ohno, F. Matsukura, J. Cibert and D. Ferrand, Science 287, 1019 (2000).

[12] A. T. Hindmarch and B. J. Hickey, Phys. Rev. Lett. 91, 116601 (2003).

[13] M. N. Baibich, J. M. Broto, A. Fert, F. Nguyen Van Dau, F. Petroff, P. Eitenne, G. Creuzet, A. Friedrich, and J. Chazelas, Phys. Rev. Lett. 61, 2472 (1988).

[14] G. Binasch, P. Grünberg, F. Saurenbach and W. Zinn, Phys. Rev. B 39, 4828 (1989).

[15] Shih-Jye Sun and Hsiu-Hau Lin, cond-mat/03xxxxx (unpublished). (2003).

[16] Shih-Jye Sun, Wei-Chun Lu, and Hsiung Chou, Physica B 324, 286 (2002).

[17] S. Doniach, E. H. Sondheimer, the chaper 7 in Green's Functions For Solid State Physicists, 1998. 